\title{
COLORING OF GRAPHS AVOIDING BICOLORED PATHS OF A FIXED LENGTH
}

\author{
ALAITTIN KIRTIŞOĞLU AND LALE ÖZKAHYA
}

\begin{abstract}
The problem of finding the minimum number of colors to color a graph properly without containing any bicolored copy of a fixed family of subgraphs has been widely studied. Most well-known examples are star coloring and acyclic coloring of graphs [9], where bicolored copies of $P_{4}$ and cycles are not allowed, respectively. In this paper, we introduce a variation of these problems and study proper coloring of graphs not containing a bicolored path of a fixed length and provide general bounds for all graphs. A $P_{k}$-coloring of an undirected graph $G$ is a proper vertex coloring of $G$ such that there is no bicolored copy of $P_{k}$ in $G$, and the minimum number of colors needed for a $P_{k}$-coloring of $G$ is called the $P_{k}$-chromatic number of $G$, denoted by $s_{k}(G)$. We provide some bounds on $s_{k}(G)$ for all graphs, in particular, proving that for any graph $G$ with maximum degree $d \geq 2$, and $k \geq 4, s_{k}(G)=O\left(d^{\frac{k-1}{k-2}}\right)$. Moreover, we find the exact values for the $P_{k}$-chromatic number of the products of some cycles and paths for $k=5,6$.
\end{abstract}

\section{INTRODUCTION}

The proper coloring problem on graphs seeks to find colorings on vertices with minimum number of colors such that no two neighbors receive the same color. There has been studies introducing additional conditions to proper coloring, such as also forbidding 2-colored copies of some particular graphs. In particular, star coloring problem on a graph $G$ asks to find the minimum number of colors in a proper coloring forbidding a 2-colored $P_{4}$, called the star-chromatic number $\chi_{s}(G)[9]$. Similarly, acyclic chromatic number of a graph $G, a(G)$, is the minimum number of colors used in a proper coloring not having any 2-colored cycle, also called acyclic coloring of $G$ [9]. Both, the star coloring and acyclic coloring problems are shown to be NP-complete in [2] and [14], respectively.

These two problems have been studied widely on many different families on graphs such as product of graphs, particularly grids and hypercubes. In this paper, we introduce a variation of these problem and study proper coloring of graphs not containing a bicolored (2-colored) path of a fixed length and provide general bounds for all graphs. The $P_{k}$-coloring of an undirected graph $G$, where $k \geq 4$, is a proper vertex coloring of $G$ such that there is no bicolored copy of $P_{k}$ in $G$, and the minimum number of colors needed for a $P_{k}$-coloring of $G$ is called the $P_{k}$-chromatic number of $G$, denoted by $s_{k}(G)$. A special case of this coloring is the star-coloring, when $k=4$, introduced by Grünbaum [9]. Hence, $\chi_{S}(G)=s_{4}(G)$ and all of the bounds on $s_{k}(G)$ in Section 2 apply to star chromatic number using $k=4$.

If a graph does not contain a bicolored $P_{k}$, then it does not contain any bicolored cycle from the family $\mathcal{C}_{k}=\left\{C_{i}: i \geq k\right\}$. Thus, as the star coloring problem is a strengthening of the acyclic coloring problem, a $P_{k}$-coloring is also a coloring avoiding a bicolored member from $\mathcal{C}_{k}$. We call such a coloring, a $\mathcal{C}_{k}$-coloring, where the minimum number of colors needed for such a coloring of a graph $G$ is called $\mathcal{C}_{k}$-chromatic number of $G$, denoted by $a_{k}(G)$. By this definition, we have

Date: December 9, 2020. 
$a_{3}(G)=a(G)$. In Section 2, we provide a lower bound for the $\mathcal{C}_{k}$-chromatic number of graphs as well.

Our results comprise lower bounds on these colorings and an asymptotic upper bound for general graphs. Moreover, some exact results are presented. In Section 2, we provide lower bounds on $s_{k}(G)$ and $a_{k}(G)$ for any graph $G$. Moreover, we show that for any graph $G$ with maximum degree $d \geq 2$, and $k \geq 4, s_{k}(G)=O\left(d^{\frac{k-1}{k-2}}\right)$. Finally, in Section 3, we present exact results on the $P_{5}$-coloring and $P_{6}$-coloring for the products of some paths and cycles.

1.1. Related Work. Acyclic coloring was also introduced in 1973 by Grünbaum [9] who proved that a graph with maximum degree 3 has an acyclic coloring with 4 colors. The following bounds obtained in [3] are the best available asymptotic bounds for the acyclic chromatic number, that are obtained using the probabilistic method.

$$
\Omega\left(\frac{d^{\frac{4}{3}}}{(\log d)^{\frac{1}{3}}}\right)=a(G)=O\left(d^{\frac{4}{3}}\right) .
$$

Recently, there have been some improvements in the constant factor of the upper bound in [15], [16], [8], by using the entropy compression method. Similar results for the star chromatic number of graphs are obtained in [7], showing $\chi_{s}(G) \leq\left\lceil 20 d^{3 / 2}\right\rceil$ for any graph $G$ with maximum degree $d$. In Section 2.1, we generalize these results to $P_{k}$-coloring of graphs.

The star chromatic number and acyclic chromatic number of products of graphs have been studied widely. In [7], various bounds on the star chromatic number of some graph families such as hypercube, grid, tori are obtained, providing exact values for 2-dimensional grids, trees, complete bipartite graphs, cycles, outer planar graphs. More recent results on the acyclic coloring of grid and tori can be found in [1] and [10]. Similarly, the acyclic chromatic number of the grid and hypercube is studied in [6]. Moreover, [11, 12, 13] investigate the acyclic chromatic number for products of trees, products of cycles and Hamming graphs. For some graphs, finding the exact values of these chromatic numbers have been a longstanding problem, such as the hypercube.

\section{General Bounds}

We obtain lower bounds on $s_{k}(G)$ and $a_{k}(G)$ by using the theorem of Erdős and Gallai below.

Theorem 1. [4] For a graph $G$ on $n$ vertices, if the number of edges is more than

(1) $\frac{1}{2}(k-2) n$, then $G$ contains $P_{k}$ as a subgraph,

(2) $\frac{1}{2}(k-1)(n-1)$, then $G$ contains a member of $\mathcal{C}_{k}$ as a subgraph,

for any $P_{k}$ with $k \geq 2$, and for any $\mathcal{C}_{k}$ with $k \geq 3$.

As also observed in [7] for the star coloring, in a $P_{k}$-coloring of a graph, the subgraphs induced by any two color classes are $P_{k}$-free. Using this observation together with Theorem 1 , we obtain the results in Theorems 2 and 3.

Theorem 2. For any graph $G=(V, E)$, let $|V|=n$ and $|E|=m$. Then, $s_{k}(G) \geq \frac{2 m}{n(k-2)}+1$, for any $k \geq 2$.

Proof. Let $s_{k}(G)=x$ and for $1 \leq i \leq x, V_{i}$ be the set of vertices whose color is $i$ in a $P_{k}$-coloring of $\mathrm{G}$ using $x$ colors. By the definition of $P_{k}$-coloring, the subgraph of $G$ induced by $V_{i} \cup V_{j}$ does 
not contain $P_{k}$ for any $1 \leq i<j \leq x$. Let $E_{i, j}$ be the set of edges covered by vertices in the set $V_{i} \cup V_{j}$. Then,

$$
\left|E_{i, j}\right| \leq \frac{1}{2}(k-2)\left|V_{i} \cup V_{j}\right|
$$

by Theorem 1 . For two distinct pairs $\left(i_{1}, j_{1}\right)$ and $\left(i_{2}, j_{2}\right)$ with $1 \leq i_{1}<j_{1} \leq x$ and $1 \leq i_{2}<j_{2} \leq x$, $E_{i_{1}, j_{1}} \cap E_{\left(i_{2}, j_{2}\right)}=\emptyset$. It also holds that

$$
\sum_{(i, j)}\left|E_{i, j}\right|=m \leq \sum_{(i, j)} \frac{1}{2}(k-2)\left(\left|V_{i}\right|+\left|V_{j}\right|\right) \leq \frac{1}{2}(k-2)(x-1) \sum_{\ell=1}^{x} V_{\ell}=\frac{n}{2}(k-2)(x-1) .
$$

The last inequality follows from the fact that each color $1 \leq \ell \leq x$ appears $(x-1)$ times over pairwise distinct pairs of colors and $\sum_{\ell=1}^{x} V_{\ell}=n$.

Theorem 3. For any graph $G=(V, E)$, let $|V|=n,|E|=m$ and $\Delta=4 n(n-1)-\frac{16 m}{k-1}+1$. Then, $a_{k}(G) \geq \frac{1}{2}(2 n+1-\sqrt{\Delta})$, for any $k \geq 3$.

Proof. Let $a_{k}(G)=x$ and for $1 \leq i \leq x, V_{i}$ be the set of vertices whose color is $i$ in a $k$-acyclic coloring of $G$ using $x$ colors. By the same ideas as in the proof of Theorem 2 and by Theorem 1, we have

$$
m \leq \sum_{(i, j)} \frac{1}{2}(k-1)\left(\left|V_{i}\right|+\left|V_{j}\right|-1\right)=(k-1)[2 n(x-1)-x(x-1)],
$$

which gives $0 \geq x^{2}-(2 n+1) x+\left(2 n+\frac{4 m}{k-1}\right)$. Let $\Delta=4 n^{2}-4 n-\frac{16 m}{k-1}+1$. We note that $\Delta \geq 1$, since $k \geq 3$ and $m \leq \frac{n(n-1)}{2}$. Thus, we have $x \geq \frac{1}{2}(2 n+1-\sqrt{\Delta})$.

2.1. Asymptotic Results. In this subsection, we obtain an asymptotic upper bound for the $P_{k^{-}}$ chromatic number of any graph on $n$ vertices and maximum degree $d$. Our proof relies on Lovász Local Lemma, for which we provide some preliminary details as follows. An event $A_{i}$ is mutually independent of a set of events $\left\{B_{i} \mid i=1,2 \ldots, n\right\}$ if for any subset $\mathcal{B}$ of events or their complements contained in $\left\{B_{i}\right\}$, we have $\operatorname{Pr}\left[A_{i} \mid \mathcal{B}\right]=\operatorname{Pr}\left[A_{i}\right]$. Let $\left\{A_{1}, A_{2}, \ldots, A_{n}\right\}$ be events in an arbitrary probability space. A graph $G=(V, E)$ on the set of vertices $V=\{1,2, \ldots, n\}$ is called a dependency graph for the events $A_{1}, A_{2}, \ldots, A_{n}$ if for each i, $1 \leq i \leq n$, the event $A_{i}$ is mutually independent of all the events $\left\{A_{j} \mid(i, j) \notin E\right\}$.

Theorem 4 (General Lovasz Local Lemma). [5] Suppose that $H=(V, E)$ is a dependency graph for the events $A_{1}, A_{2}, \ldots, A_{n}$ and suppose there are real numbers $y_{1}, y_{2}, \ldots, y_{n}$ such that $0 \leq y_{i} \leq 1$ and

$$
\operatorname{Pr}\left[A_{i}\right] \leq y_{i} \prod_{(i, j) \in E}\left(1-y_{j}\right) \quad \text { for all } 1 \leq i \leq n .
$$

Then $\operatorname{Pr}\left[\bigwedge_{i=1}^{n} \bar{A}_{i}\right] \geq \prod_{i=1}^{n}\left(1-y_{i}\right)$. In particular, with positive probability no event $A_{i}$ holds.

We use Theorem 4 in the proof of the following upper bound.

Theorem 5. Let $G$ be any graph with maximum degree $d$. Then $s_{k}(G) \leq\left\lceil 6 \sqrt{10} d^{\frac{k-1}{k-2}}\right\rceil$, for any $k \geq 4$ and $d \geq 2$.

Proof. Assume that $x=\left\lceil a d^{\frac{k-1}{k-2}}\right\rceil$ and $a=6 \sqrt{10}$. Let $f: V \mapsto\{1,2, \ldots, x\}$ be a random vertex coloring of $G$, where for each vertex $v \in V$, the color $f(v) \in\{1,2, \ldots, x\}$ is chosen uniform randomly. It suffices to show that with positive probability $f$ does not produce a bicolored $P_{k}$.

Below are the types of probabilistic events that are not allowed: 
- Type I: For each pair of adjacent vertices $u$ and $v$ of $G$, let $A_{u, v}$ be the event that $f(u)=$ $f(v)$.

- Type II: For each $P_{k}$ called $P$, let $A_{P}$ be the event that $P$ is colored properly with two colors.

By definition of our coloring, none of these events are allowed to occur. We construct a dependency graph $H$, where the vertices are the events of Types I and II, and use Theorem 4 to show that with positive probability none of these events occur. For two vertices $A_{1}$ and $A_{2}$ to be adjacent in $H$, the subgraphs corresponding to these events should have common vertices in $G$. The dependency graph of the events in is called $H$, where the vertices are the union of the events We call a vertex of $H$ of Type $i$ if it corresponds to an event of Type i.

Observation 1. For any vertex $v$ in $G$, there are at most

- d pairs $\{u, v\}$ associated with an event of Type $I$, and

- $\frac{k+1}{2} d^{k-1}$ copies of $P_{k}$ containing $v$, associated with an event of Type II.

Proof. The first observation follows from the fact that $\Delta(G)=d$. To see the second observation, let us label the vertices of a $P_{k}$ containing $v$ as $x_{1}, x_{2}, \ldots, x_{k}$. The maximum number of $P_{k}$ 's with $x_{i}=v$ is $d^{k-1}$. Considering that $1 \leq i \leq k$, there are at most $\left\lceil\frac{k}{2}\right\rceil d^{k-1}$ copies of $P_{k}$ containing $v$ considering the symmetric positions on the path.

Lemma 6. The $(i, j)^{\text {th }}$ entry of the following matrix shows an upper bound on the number of vertices of type $j$ that are adjacent to a vertex of type $i$ in $H$.

\begin{tabular}{|c|c|c|}
\hline & $I$ & $I I$ \\
\hline$I$ & $2 d$ & $(k+1) d^{k-1}$ \\
\hline$I I$ & $k d$ & $\frac{k}{2}(k+1) d^{k-1}$ \\
\hline
\end{tabular}

Proof. Consider a vertex $A_{u, v}$ in $H$ for the first row. Since, this vertex may be adjacent to events $A_{u, z}$ and $A_{v, x}$ for some $x, z \in V(G)$, there are at most $2 d$ such events of Type I. Similarly, $A_{u, v}$ may be adjacent to events $A_{P}$, where $P$ is a $P_{k}$ containing $u$ or $v$. There are at most $(k+1) d^{k-1}$ such events. For the second row, a path $P$ that is a copy of $P_{k}$ may have $k d$ edges intersecting it. Similarly, there may be at most $(k+1) d^{k-1} / 2$ other $P_{k}$ 's containing some particular vertex of $P$.

Observation 2. The probabilities of the events are

- $\operatorname{Pr}\left(A_{u, v}\right)=\frac{1}{x}$ for an event of type I, and

- $\operatorname{Pr}\left(A_{P}\right)=\frac{1}{x^{k-2}}$ for an event of type II.

To apply Theorem 4, we choose the weights $y_{i}$ as below:

$$
y_{1}=\frac{1}{3 d}, \quad y_{2}=\frac{1}{2(k+1) d^{k-1}} .
$$

Below are the conditions that are to be satisfied for (1) to hold.

$$
\begin{gathered}
\frac{1}{x} \leq \frac{1}{3 d}\left(1-\frac{1}{3 d}\right)^{2 d}\left(1-\frac{1}{2(k+1) d^{k-1}}\right)^{(k+1) d^{k-1}} \\
\frac{1}{x^{k-2}} \leq \frac{1}{2(k+1) d^{k-1}}\left(1-\frac{1}{3 d}\right)^{k d}\left(1-\frac{1}{2(k+1) d^{k-1}}\right)^{\frac{k}{2}(k+1) d^{k-1}}
\end{gathered}
$$


Since $(1+x)^{n} \geq 1+n x$ for $x \geq-1$ and any nonnegative integer $n$, it is sufficient to verify the following to satisfy (2), and we observe that it holds when $a=6 \sqrt{10} \geq 18$ and $k \geq 4$.

$$
\frac{1}{a d^{\frac{k-1}{k-2}}} \leq \frac{1}{3 d}\left(1-\frac{2 d}{3 d}\right)\left(1-\frac{(k+1) d^{k-1}}{2(k+1) d^{k-1}}\right)=\frac{1}{18 d}
$$

We can rewrite (3) as below.

$$
\frac{1}{a} \leq\left(\frac{1}{2(k+1)}\right)^{\frac{1}{k-2}}\left(1-\frac{1}{3 d}\right)^{\frac{k d}{k-2}}\left(1-\frac{1}{2(k+1) d^{k-1}}\right)^{\frac{k(k+1) d^{k-1}}{2(k-2)}}
$$

Since $(1+x)^{n} \geq 1+n x$ for $x \geq-1$ and any nonnegative integer $n$, it is sufficient to verify the following to satisfy (3). We omit the use of ceiling for simplicity.

$$
\frac{1}{a} \leq\left(\frac{1}{2(k+1)}\right)^{1 / k-2}\left(1-\frac{k}{3(k-2)}\right)\left(1-\frac{k}{4(k-2)}\right)
$$

Since all factors on the right are decreasing for $k \geq 4$, (3) is verified.

\section{Coloring of Products of Paths and Cycles}

The cartesian product of two graphs $G=(V, E)$ and $G^{\prime}=\left(V^{\prime}, E^{\prime}\right)$ is shown by $G \square G^{\prime}$ and its vertex set is $V \times V^{\prime}$. For any vertices $x, y \in V$ and $x^{\prime}, y^{\prime} \in V^{\prime}$, there is an edge between $(x, y)$ and $\left(x^{\prime}, y^{\prime}\right)$ in $G \square G^{\prime}$ if and only if either $x=y$ and $x^{\prime} y^{\prime} \in E^{\prime}$ or $x^{\prime}=y^{\prime}$ and $x y \in E$. For simplicity, we let $G(n, m)$ denote the product $P_{n} \square P_{m}$.

\section{1. $P_{5}$-coloring of Graphs.}

\section{Theorem 7 .}

$$
s_{5}\left(P_{3} \square P_{3}\right)=s_{5}\left(C_{3} \square C_{3}\right)=s_{5}\left(C_{3} \square C_{4}\right)=s_{5}\left(C_{4} \square C_{4}\right)=4 .
$$

Proof. We start by showing that $s_{5}\left(P_{3} \square P_{3}\right) \geq 4$. Assume that there is a coloring of $P_{3} \square P_{3}$ using colors $\{a, b, c\}$ only. We want to show that each color appears exactly twice in any consecutive columns. Note that each color appears at most 3 times in consecutive columns. If a color, say $a$, appears 3 times, then by pigeonhole principle, a color, say $c$, appears exactly once on these consecutive columns. In this case, the vertices colored $a$ and $b$ contain a bicolored $P_{5}$. Hence, each color is used exactly twice and all colors appear in any consecutive columns.

Without loss of generality, suppose that $a$ is used twice in a column. Then, in a consecutive column, either $b$ or $c$ is used twice, which is impossible in a proper coloring using $\{a, b, c\}$ only. Thus, each column has colors $a, b, c$ exactly once. This implies that any coloring of $P_{3} \square P_{3}$ using three colors have to be colored as shown below. According to this property, if the vertex at the center of $P_{3} \square P_{3}$ has, say color $a$, then some pair of vertices at opposing corners have color $a$ as well. As we see in (4), however the remaining vertices are colored, there is always a bicolored $P_{5}$, thus $s_{5}\left(P_{3} \square P_{3}\right) \geq 4$.

$\begin{array}{lllllll}a & b & c & 1 & 2 & 3 & 4 \\ c & a & b & 2 & 1 & 4 & 3 \\ b & c & a & 3 & 4 & 1 & 2 \\ & & & 4 & 3 & 2 & 1\end{array}$

Since $C_{3} \square C_{3}, C_{3} \square C_{4}$ and $C_{4} \square C_{4}$ contain $P_{3} \square P_{3}$ as a subgraph, this shows that at least 4 colors are needed to color these graphs. Such a coloring can be obtained as in (4) by taking the first three or four rows/columns depending on the change in the grid dimension. 
Theorem 8. $s_{5}(G(n, m))=4$ for all $n, m \geq 3$.

Proof. Note that $4=s_{5}(G(3,3)) \leq s_{5}(G(n, m))$ for all $m, n \geq 3$. Since there exists some integer $k$ for which $3 k \geq n, m$ and $G(n, m)$ is a subgraph of $G(3 k, 3 k), s_{5}(G(n, m)) \leq s_{5}(G(3 k, 3 k))$ for some $k$. Hence, we show that $s_{5}(G(3 k, 3 k))=4$. In Theorem 7, a $P_{5}$-coloring of $C_{3} \square C_{3}$ is given by the upper left corner of the coloring in (4) by using 4 colors. By repeating this coloring of $C_{3} \square C_{3} k$ times in $3 k$ rows, we obtain a coloring of $G(3 k, 3)$. Then repeating this colored $G(3 k, 3) k$ times in $3 k$ columns, we obtain a $P_{5}$-coloring of $G(3 k, 3 k)$ using 4 colors. There exists no bicolored $P_{5}$ in this coloring.

In the following, we generalize the previous cases by making use of the well-known result below.

Theorem 9 (Sylvester, [17]). If $r, s>1$ are relatively prime integers, then there exist $\alpha, \beta \in \mathbb{N}$ such that $t=\alpha r+\beta s$ for all $t \geq(r-1)(s-1)$.

Theorem 10. Let $p, q \geq 3$ and $p, q \neq 5$. Then $s_{5}\left(C_{p} \square C_{q}\right)=4$.

Proof. The lower bound follows from Theorem 7. By Theorem 9, $p$ and $q$ can be written as a linear combination of 3 and 4 using nonnegative coefficients. By using this, we are able to tile the $p \times q$-grid of $C_{p} \square C_{q}$ using these blocks of $3 \times 3,3 \times 4,4 \times 3$, and $4 \times 4$ grids. Recall that the coloring pattern in (4) also provides a $P_{5}$-coloring of smaller grids listed above by using the upper left portion for the required size. Therefore, using these coloring patterns on the smaller blocks of the tiling yields a $P_{5}$-coloring of $C_{p} \square C_{q}$.

Corollary 11. Let $i, j \geq 3$ and $i, j \neq 5$. Then, $s_{5}\left(P_{i} \square C_{j}\right)=4$.

Proof. Since $P_{i} \square P_{j}$ is a subgraph of $P_{i} \square C_{j}$, Theorem 8 gives the lower bound. By Theorem 10, we have the equality.

\section{2. $P_{6}$-coloring of Graphs.}

Theorem 12. For $n, m \geq 4, s_{6}(G(4,4))=4$.

Proof. Since $s_{6}(G(4,4)) \leq s_{5}(G(4,4))=4$, we prove $s_{6}(G(4,4)) \geq 4$. Assume that $f$ is a coloring of $G(4,4)$ using the colors $\{1,2,3\}$ only. We consider possible colorings on the $C_{4}$ at the center of the grid, call it $C$.
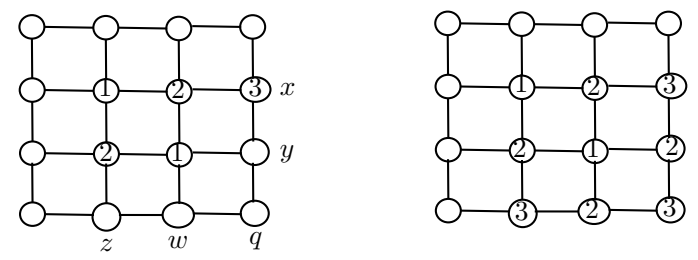

Figure 1. Possible colorings in Case 1

Case 1: $C$ is bicolored. Assume that $C$ has only two colors, 1 and 2. Then, either $x$ or $y$ shown in Figure 1 has color 3. Without loss of generality, assume that $f(x)=3$. This implies $f(y)=2$. To avoid a bicolored $P_{6}$, we have $f(q)=3$. This implies that $f(w)=2$, and therefore $f(z)=3$ so that $V(C) \cup\{z, w\}$ is not bicolored. However, this yields a bicolored $P_{7}$ as shown in Figure 1.

Case 2: $C$ has all three colors. We assume that the repeating color on $C$ is 1 . 
Case 2.a: Color 1 is also used on the pair of vertices in opposing corners as in Figure 2a. Note that $x$ and $y$ cannot have the same color, otherwise there is a bicolored $P_{6}$. Same holds for $w$ and $z$. Hence, both 2 and 3 appear as colors on the pairs $\{x, y\}$ and $\{w, z\}$, yielding a bicolored $P_{6}$.

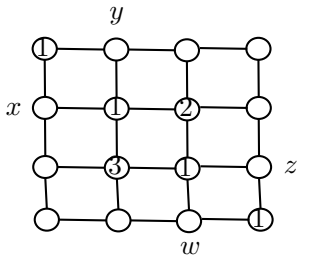

(A)

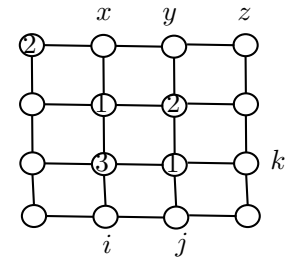

(B)

Figure 2. Possible colorings in Case 2.

Case 2.b: Color 1 is not used on both of the vertices in opposing corners as in Figure 2a. Without loss of generality, assume that one of the vertices at the corners is colored 2 as in Figure $2 \mathrm{~b}$. This case is also symmetric to the case when this color is 3 . This implies that $f(x)=3$ and $f(y)=1$ yielding a bicolored $P_{5}$. To avoid a bicolored (with colors 1 and 3 ) $P_{6}$, it is necessary that $f(j)=f(k)=f(z)=2$. However, this produces a bicolored $P_{6}$ shown in Figure 2b.

Corollary 13. $s_{6}(G(n, m))=4$ for all $n, m \geq 4$.

Proof. By Theorem 8, we have $s_{6}(G(n, m)) \leq s_{5}(G(n, m))=4$. And, by Theorem 12, we have equality.

Corollary 14. $s_{6}\left(C_{m} \square C_{n}\right)=4$ for all $m, n \geq 4$ and $m, n \neq 5$.

Proof. By the definition of $P_{k}$-coloring and Theorem 10, $s_{6}\left(C_{m} \square C_{n}\right) \leq s_{5}\left(C_{m} \square C_{n}\right)=4$ for all $m, n \geq 3$ and $m, n \neq 5$. Since $G(4,4)$ is a subgraph of $C_{m} \square C_{n}$ for all $m, n \geq 4$ and by Corollary 13 , $s_{6}\left(C_{m} \square C_{n}\right) \geq s_{6}(G(4,4))=4$.

\section{Conclusions}

In this paper, we introduced a variation of the star coloring problem by forbidding a family different than double stars to be bicolored, such as paths of a fixed length and cycles that are longer than some fixed $k$. We could study only one family of graphs and investigating these colorings for other graph families, such as higher dimensional grids and tori would be interesting. In the following, we summarize some questions that remain open.

- The method used in Theorem 10 for $s_{5}\left(C_{p} \square C_{q}\right)$ could not be generalized to the case when either $p$ or $q$ is 5 .

- We observe that $s_{k}(G(2, m))=3$ for all $m \geq 3, k \geq 5$, and $s_{k}(G(3, m))=3$ for all $m \geq 3$ and $k \geq 6$. By the definition of $P_{k}$-coloring, we need at least three colors and a 3 -coloring is possible as shown below.

$\begin{array}{llllllllllllll}1 & 3 & 2 & 1 & 3 & 2 & \ldots & 1 & 2 & 3 & 1 & 2 & 3 & \ldots \\ 2 & 1 & 3 & 2 & 1 & 3 & \ldots & 2 & 3 & 1 & 2 & 3 & 1 & \ldots \\ 1 & 2 & 3 & 1 & 2 & 3 & \ldots\end{array}$

One could possibly find $s_{k}(G(n, m))$ and $s_{k}\left(C_{p} \square C_{q}\right)$ for all $k$ using methods similar to ours. - It would be interesting to obtain a bound similar to Theorem 5 for $a_{k}(G)$ of any graph $G$. 


\section{ACKNOWLEDGEMENTS}

The research of the second author was supported in part by the BAGEP Award of the Science Academy.

\section{REFERENCES}

1. S Akbari, M Chavooshi, M Ghanbari, and S Taghian, Star coloring of the cartesian product of cycles, arXiv preprint arXiv:1906.06561 (2019). 1.1

2. Michael O Albertson, Glenn G Chappell, Hal A Kierstead, André Kündgen, and Radhika Ramamurthi, Coloring with no 2-colored $p_{-} 4$ 's, the electronic journal of combinatorics (2004), R26-R26. 1

3. Noga Alon, Colin Mcdiarmid, and Bruce Reed, Acyclic coloring of graphs, Random Structures \& Algorithms 2 (1991), no. 3, 277-288. 1.1

4. Paul Erdős and Tibor Gallai, On maximal paths and circuits of graphs, Acta Mathematica Academiae Scientiarum Hungarica 10 (1959), no. 3-4, 337-356. 1

5. Paul Erdős and László Lovász, Problems and results on 3-chromatic hypergraphs and some related questions, in Infinite and Finite Sets (A. Hajnal et al., eds), North-Holland, Amsterdam, 1975. 4

6. Guillaume Fertin, Emmanuel Godard, and André Raspaud, Acyclic and k-distance coloring of the grid, (2003). 1.1

7. Guillaume Fertin, André Raspaud, and Bruce Reed, Star coloring of graphs, Journal of Graph Theory 47 (2004), no. 3, 163-182. 1.1, 2

8. Daniel Gonçalves, Mickaël Montassier, and Alexandre Pinlou, Entropy compression method applied to graph colorings, arXiv preprint arXiv:1406.4380 (2014). 1.1

9. Branko Grünbaum, Acyclic colorings of planar graphs, Israel journal of mathematics 14 (1973), no. 4, 390-408. (document), 1, 1.1

10. Tianyong Han, Zehui Shao, Enqiang Zhu, Zepeng Li, and Fei Deng, Star coloring of cartesian product of paths and cycles., Ars Comb. 124 (2016), 65-84. 1.1

11. Robert E Jamison and Gretchen L Matthews, Acyclic colorings of products of cycles, Bulletin of the Institute of Combinatorics and its Applications 54 (2008), 59-76. 1.1

12. On the acyclic chromatic number of hamming graphs, Graphs and Combinatorics 24 (2008), no. 4, 349-360. 1.1

13. Robert E Jamison, Gretchen L Matthews, and John Villalpando, Acyclic colorings of products of trees, Information Processing Letters 99 (2006), no. 1, 7-12. 1.1

14. Alexandr V Kostochka, Upper bounds of chromatic functions of graphs, Doct. Thesis, Novosibirsk, 1978. 1

15. Sokol Ndreca, Aldo Procacci, and Benedetto Scoppola, Improved bounds on coloring of graphs, European Journal of Combinatorics 33 (2012), no. 4, 592-609. 1.1

16. Jean-Sébastien Sereni and Jan Volec, A note on acyclic vertex-colorings, arXiv preprint arXiv:1312.5600 (2013). 1.1

17. James J Sylvester et al., Mathematical questions with their solutions, Educational times 41 (1884), no. 21, 171-178. 9

Hacettepe University, Department of Mathematics, Beytepe 06810 Ankara, Turkey

Email address: alaittinkirtisoglu@gmail.com

Hacettepe University, Department of Computer Engineering, Beytepe 06810 Ankara, Turkey

Email address: ozkahya@cs.hacettepe.edu.tr 